TAIWANESE JOURNAL OF MATHEMATICS

Vol. 12, No. 7, pp. 1751-1756, October 2008

This paper is available online at http://www.tjm.nsysu.edu.tw/

\title{
AN EXTENSION OF JUNGCK AND SESSA RESULT
}

\author{
Arjamand Bano
}

\begin{abstract}
The purpose of this paper is to extend the work of Jungck and Sessa [4] from single-valued maps to multivalued maps.
\end{abstract}

\section{INTRODUCTION}

Brosowski [1], Meinardus [9] and Singh [12] established some results on invariant approximation in normed spaces using fixed point theory. Jungck and Sessa [4] worked over approximation theory for setting of normed spaces. Later on, several generalizations of their results were obtained by Habiniak [2], Hicks and Humphries [3], Khan, Hussain and Thaheem [7], Latif and Bano [8] and Narang [10]. However, another important aspect of their work is still not explored and needs attention and that is the application of their results to multi-valued maps. Therefore, we have decided to extend the result of Jungck and Sessa [4] from single- valued maps.

Throughout the manuscript, the following definitions and results have been used. Let $X$ be a normed space and $M$ be its nonempty subset. We denote the families of all nonempty closed bounded and nonempty compact subsets of $X$ by $C B(X)$ and $K(X)$ respectively. Let $H$ be the Hausdorff metric on $C B(X)$ induced by the norm of $X$ which means

$$
H(A, B)=\max \left\{\sup _{a \in A} \inf _{b \in B}\|a-b\|\right\}, \sup _{a \in B} \inf _{b \in B}\|a-b\|, \text { for } A, B \text { in } C B(X),
$$

$M$ is said to be starshaped with respect to a point $q \in M$ if $(1-h) q+h x \in M$ for all $x \in M$ and all $h \in(0,1)$. Further, each convex set is necessarily starshped, but a starshaped need not be convex.

Received September 30, 2006, accepted May 5, 2007.

Communicated by Sen-Yen Shaw.

2000 Mathematics Subject Classification: 47H10, 54C60, 54H25, 55M20.

Key words and phrases: Multi-valued $\wp$-nonexpansive map, Fixed points, Common fixed points, Starshaped, Affine map, Best M-approximants, Normed spaces. 
Let $P_{M}\left(x_{0}\right)=\left\{y \in M: d\left(y, x_{0}\right)=d\left(x_{0}, M\right)\right\}$ the set of best $M$ approximation to $x_{0} . P_{M}\left(x_{0}\right)$ is always bounded subset of $X$ and it is closed or convex if $M$ is closed or convex [1]. We adopt the following definitions for convenience. Let $\wp$ be a family of single-valued self map of $M$ and $\wp f=\left\{f^{n}: M \rightarrow M, n \geq 0\right\}\left(f^{0}=I\right)$. A map $T: M \rightarrow C B(M)$ said to be

(i) $\wp$-nonexpansive [6], if for all $x, y \in M$ there exists $f, g \in \wp$ such that

$$
H(T x, T y) \leq\|f x-g x\| ;
$$

(ii) $\wp$-contraction [6], if for all $x, y \in M$ there exists a real number $h \in(0,1)$ such that

$$
H(T x, T y) \leq h\|f x-g y\| \text { for some } f, g \in \wp ;
$$

(iii) $\wp_{f}$-nonexpansive, if for each $x, y \in M$ there exists $n, m \geq 0$ such that

$$
H(T x, T y) \leq\left\|f^{n} x-f^{m} y\right\| ;
$$

We denote by $F(T)$ and $F(f)(F(\wp))$ the set of all fixed points of $T$ and $f$ (the set of all common fixed points of $\wp)$. Also we say that $\wp$ and $T$ commute, if for each $f \in \wp$ commutes with $T$.

In 1995 Jungck and Sessa [4] obtained the following generalization of Sahab, Khan and Sessa result [11].

Theorem 1.1. Let $M$ be compact subset of a normed linear space, $X$ which is starshaped with respect to $q \in M$. Let $T: M \rightarrow M$ be continuous and let $\wp$ be a family of affine maps $f: M \rightarrow M$ such that $q \in F(f)$ and $f T=T f$ If for each pair $(x, y) \in M^{2}$ there exists $f, g \in \wp$ such that

$$
\|T x-T y\| \leq\|f x-g y\|,
$$

then there exists $a \in M$ such that $a=T(a)$ and $a=f(a)$ for all continuous $f \in \wp$.

We now state a theorem, which is a special case of Theorem 1 in [5].

Theorem 1.2. Let $(X, d)$ be a compact metric space. Let $\wp$ be a family of continuous self mappings of $X$ and $T: X \rightarrow K(X)$ be $\wp$-contraction multivalued map such that $T(X) \subseteq f(X)$ for all $f \in \wp$ and $\wp$ commutes with $T$. Then $F(T) \cap F(\wp) \neq \phi$. 


\section{RESULTS}

In this section Theorem 1.1 has been extended to multi-valued $\wp$-nonexpansive map.

Theorem 2.1. Let $M$ be a compact subset of a normed linear space, $X$ which is starshaped with respect to $q \in M$. Let $\wp$ be family of continuous affine maps $f: M \rightarrow M$ such that $q \in F(f)$ for all $f \in \wp$. If $T: M \rightarrow K(M)$ is multi-valued $\wp$-nonexpansive map which commutes with $f$ for all $f \in \wp$ then there exists a point $z \in M$ such that $z \in F(T)$ and also $z \in F(f)$ for all $f \in \wp$ then $F(T) \cap F(f) \neq \phi$

Proof. Consider a sequence $\left\{h_{n}\right\}$ of real numbers for which $0<h_{n}<1$ and $h_{n} \rightarrow 1$ as $n \rightarrow \infty$. For each $n$, a multi-valued map $T_{n}$ is defined by setting

$$
T_{n}(x)=h_{n} T x+\left(1-h_{n}\right) q, \quad(x \in M) .
$$

Now we prove that for each $n \geq 1, T_{n}$ maps $M$ into $K(M)$. Indeed we show that $T_{n}(x)$ is compact for all $x \in M$. Let $x_{k} \in T_{n}(x), k=1,2, \ldots$, be a sequence of $T_{n}(x)$. We get $x_{k}=h_{n} u_{k}+\left(1-h_{n}\right) q$ for some $u_{k} \in T(x)$ and, by using the compactness of $T(x)$ there exists a suitable subsequence $\left\{u_{k(p)}, p=1,2, \ldots,\right\}$ such that $u_{k(p)} \rightarrow u \in T(x)$ By setting $x_{0}=h_{n} u+\left(1-h_{n}\right) q$, we have that there is a subsequence $\left\{x_{k(p)}, k=1,2, \ldots,\right\}$ of $x_{k}$, such that $x_{k(p)}=h_{n} u_{k(p)}+\left(1-h_{n}\right) q \rightarrow$ $x_{0}$. This proves that $T_{n}(x)$ is compact.

Next we show that $T_{n}$ is $\wp$-contraction and commutes with $\wp$. Let $x, y \in M$ and $u_{x} \in T_{n}(x)$ then $u_{x}=h_{n} v_{x}+\left(1-h_{n}\right) q$ for some $v_{x} \in T(x)$. Since for all $f, g \in \wp, T$ is multivalued $\wp$-nonexpansive so there exists $v_{y} \in T(y)$ for all $y \in M$ such that

$$
d\left(v_{x}, v_{y}\right) \leq H(T x, T y) .
$$

Put $u_{y}=h_{n} v_{y}+\left(1-h_{n}\right) q$ then $v_{y} \in T(y)$ and

$$
d\left(u_{x}, u_{y}\right)=h_{n} d\left(v_{x}, v_{y}\right) \leq h_{n} H(T x, T y) .
$$

so by the $\wp$-nonexpansiveness of $T$ we have

$$
d\left(u_{x}, u_{y}\right) \leq h_{n} d(f(x), g(y)) .
$$

It follows that

$$
\sup _{u_{x} \in T_{n}(x)} d\left(u_{x}, T_{n}(y)\right) \leq d\left(u_{x}, u_{y}\right) \leq h_{n} d(f(x), g(y)) .
$$

The same argument concludes that

$$
\sup _{u_{y} \in T_{n}(y)} d\left(u_{y}, T_{n}(x)\right) \leq h_{n} d(f(x), g(y)) .
$$


Hence

$$
H\left(T_{n} x, T_{n} y\right) \leq h_{n}\|f x-g y\|, \forall f, g \in \wp
$$

Which proves that each $T_{n}$ is $\wp$-contraction for each $x \in M$. Moreover, since $T$ commutes with $\wp$ and each $f \in \wp$ is affine so for each $x \in M$,

$$
\begin{aligned}
T_{n} f x & =h_{n} T f x+\left(1-h_{n}\right) f q \\
& =h_{n} f T x+\left(1-h_{n}\right) f q \\
& =f\left(h_{n} T x+\left(1-h_{n}\right) q\right) \\
& =f T_{n} x
\end{aligned}
$$

Thus each $T_{n}$ commutes with $f$. As all the conditions of Theorem 1.2 are satisfied hence $x_{n} \in M$ such that $x_{n} \in T_{n} x_{n}$ and $x_{n}=f x_{n}$ for all $f \in \wp$. So by the definition of $T_{n} x_{n}$ there is some $w_{n} \in T x_{n}$ such that

$$
x_{n}=h_{n} w_{n}+\left(1-h_{n}\right) q
$$

Since $\left\{x_{n}\right\}$ is a sequence in a compact set $M$, there exists a subsequence $\left\{x_{n_{i}}\right\}$. with $x_{n_{i}} \rightarrow z \in M$ As $z=\lim _{i} x_{n_{i}}=\lim _{i} h_{n_{i}} w_{n_{i}}+\lim _{i}\left(1-h_{n_{i}}\right) q$ and that $h_{n_{i}} \rightarrow 1$ then it follows that $z \in T z$, which implies that $z \in F(T)$. Further, if $f \in \wp$ is continuous, then it follows that

$$
z=\lim _{i} x_{n_{i}}=f\left(\lim _{i} x_{n_{i}}\right)=f(z)
$$

which implies that $z=f(z)$

Corollary 2.2. Let $M$ be a compact subset of a normed space $X$ which is starshaped with respect to $q \in M$. Let $f$ be self affine map of $M$ with $q \in F(f)$. If $T: M \rightarrow K(M)$ is multi-valued $\wp_{f}$-nonexpansive map and commute with $T$ then $F(T) \neq \phi$. Moreover, if $f$ is continuous, then $F(T) \cap F\left(\wp_{f}\right) \neq \phi$

Proof. Let $\wp_{f}=\left\{f^{n}: n \geq 0\right\}\left\{f^{0}=I\right\}$ For each $n, f^{n}$ is affine, $T f^{n}=f^{n} T$ and $f^{n} q=q$ and $f^{n}: M \rightarrow M$ since $f$ has these properties. Now the proof of the corollary follows from Theorem 2.1.

\section{A Result in Best Approximation Theory}

We shall require the following Lemma, proved by Hicks and Humphries ([3], p. 221) for normed spaces.

Lemma 3.1. Let $M$ be a subset of a normed space $X$. Then, for any $x_{0} \in$ $X, P_{M}\left(x_{0}\right) \subseteq \partial M($ the boundary of $M)$. 
Theorem 3.2. Let $X$ be a normed space and $T: X \rightarrow K(X)$ be multivalued map such that $T\left(x_{0}\right)=\left\{x_{0}\right\}$ for some $x_{0} \in X$ and $f: X \rightarrow X$ be single-valued map such that $x_{0} \in F(f)$. Let $M$ be a subset of $X$ such that $T(\partial M) \subseteq M$. Suppose $T$ is $\wp_{f}$-nonexpansive on $P_{M}\left(x_{0}\right) \cup\left\{x_{0}\right\}, f$ is affine and commutes with $T$ on $P_{M}\left(x_{0}\right)$. If $P_{M}\left(x_{0}\right)$ is nonempty, compact and starshaped with respect to $q \in F(f)$ and if, $f\left(P_{M}\left(x_{0}\right)\right) \subseteq P_{M}\left(x_{0}\right)$, then $P_{M}\left(x_{0}\right) \cap F(T) \neq \phi$. Further if $f$ is continuous on $P_{M}\left(x_{0}\right)$, then $P_{M}\left(x_{0}\right) \cap F(T) \cap F\left(\wp_{f}\right) \neq \phi$.

Proof. Let $D=P_{M}\left(x_{0}\right)$ and $u \in D$. Then $u \in M$ and

$$
\left\|x_{0}-u\right\|=d\left(x_{0}, M\right) .
$$

Let $v \in T(u) \subset M$. Then we have

$$
\left\|v-x_{0}\right\| \leq H\left(T(u), T\left(x_{0}\right)\right)
$$

Using the $\wp$-nonexpansiveness of $T$, one gets

$$
H\left(T(u), T\left(x_{0}\right)\right) \leq\left\|f^{n}(u)-f^{m}\left(x_{0}\right)\right\|
$$

for some $m, n \geq 0$. As $f^{m}\left(x_{0}\right)=x_{0}$ for $m \geq 0$, and $f\left(P_{M}\left(x_{0}\right)\right) \subseteq P_{M}\left(x_{0}\right)$, so for all $n \geq 0, f^{n}(u) \in P_{M}\left(x_{0}\right)$, and

$$
\left\|v-x_{0}\right\|=d\left(x_{0}, M\right) .
$$

implies that $v \in D$ and thus $T(u) \subset D$. Therefore $T$ carries $D$ into $K(D)$.

Thus, by Corollary 2.2 the conclusion holds.

We observe that if $f=I$ the identity map on $P_{M}\left(x_{0}\right)$, then $\wp_{f}$-nonexpansive map is the usual nonexpansive map.

In this case we have the following result.

Corollary 3.3. Let $X$ be a normed space and $T: X \rightarrow K(X)$ such that $T\left(x_{0}\right)=x_{0}$ for some $x_{0} \in X . M$ be a subset of $X$ such that $T(\partial M) \subseteq M$ Let $T$ be nonexpansive map on $P_{M}\left(x_{0}\right) \cup\left\{x_{0}\right\}$. If $P_{M}\left(x_{0}\right)$ is nonempty compact, and starshaped, then $P_{M}\left(x_{0}\right) \cap F(T) \neq \phi$

\section{Remark 3.4.}

(1) Theorem 3.2 is an extension of Theorem 4 of Jungck and Sessa [4] which in turn includes the main result of Sahab, Khan and Sessa [11].

(2) Corollary 3.3 extends the main result of Singh [12]. 


\title{
REFERENCES
}

1. B. Brosowski, Fixpunktsatze in der Approximations Theorie, Mathematica(cluj), 11 (1969), 195-220.

2. L. Habiniak, Fixed point theorems and invariant approximation, J. Approx. Theory, 56 (1989), 241-244.

3. T. L. Hicks and M. D. Humphries, A note on fixed point theorems, J. Approx. Theory, 34 (1982), 221-225.

4. G. Jungck and S. Sessa, Fixed point theorems in best approximation theory, Math. Japon, 42 (1995), 249-252.

5. H. Kaneko, A Banach type fixed point theorem for multivalued mappings, $K$. $J$. Math., (1984), 163-165.

6. H. Kaneko, Single-valued and multivalued f-contraction, Boll. U.M.I., 6 (1985), 29-33.

7. A. R. Khan, N. Hussain, and A. B. Thaheem, Application of fixed point theorems to invariant approximation, J. Approx. Theory and its Appl., (2000), 48-55.

8. A. Latif and A. Bano, A result on invariant approximation, Tamkang J. Math., 33(1) (2002), 89-92.

9. G. Meinardus, Invarianze bei Linearen Approximationen, Arch. Rational Mech. Anal., 14 (1963), 301-303.

10. T. D. Narang, Applications of fixed point theorems to approximation theory, Math, Vesnik, 36 (1984), 69-75.

11. S. A. Sahab, M. S. Khan and Sessa, A result in best approximation theory, J. Approx. Theory, 55 (1988), 349-351.

12. S. P. Singh, An application of fixed point theorem to approximation theory, J. Approx. Theory, 25 (1979), 89-90.

\author{
Arjamand Bano \\ Department of Mathematics, \\ Gomal University, \\ Dera Ismail Khan, \\ Pakistan \\ E-mail: arjamandbano2002@yahoo.com
}

